\title{
Acousto-optical multiple interference switches
}

\author{
M. Beck
}

Paul-Drude-Institut für Festkörperelektronik, Hausvogteiplatz 5-7, D-10117 Berlin, Germany

M. M. de Lima, Jr.

Paul-Drude-Institut für Festkörperelektronik, Hausvogteiplatz, 5-7, D-10117 Berlin, Germany and Materials Science Institute, University of Valencia, P.O. Box 22085, E-46071 Valencia, Spain

\author{
E. Wiebicke, W. Seidel, R. Hey, and P. V. Santos ${ }^{\text {a) }}$ \\ Paul-Drude-Institut für Festkörperelektronik, Hausvogteiplatz, 5-7, D-10117 Berlin, Germany
}

(Received 14 June 2007; accepted 16 July 2007; published online 9 August 2007)

\begin{abstract}
The authors introduce an alternative approach for acousto-optical light control based on the interference of light propagating through several waveguides, each subjected to a periodic refractive index modulation induced by a surface acoustic wave. The feasibility of the concept is demonstrated by the realization of an optical switch for arbitrary time intervals with an on/off contrast ratio of 20. ㄷ 2007 American Institute of Physics. [DOI: 10.1063/1.2768889]
\end{abstract}

The acousto-optical interaction provides a wellestablished process for controlling light beams in solids. ${ }^{1}$ With the advent of integrated optics, approaches have been sought after to implement the required phase matching between the acoustic and optical waves propagating in waveguide (WG) structures. Examples are the acoustically induced coupling between neighboring WGs (Ref. 1) and the use of Umklapp scattering in photonic crystals. ${ }^{2,3}$

As an alternative approach, Gorecki et al. proposed the combination of surface acoustic waves (SAWs) with MachZehnder interferometers (MZIs) for WG modulators. ${ }^{4,5}$ Here, the strain field of a SAW impinging perpendicularly to one of the interferometer arms introduces a periodic modulation of the transmitted light. More recently, de Lima et al. ${ }^{6}$ introduced an approach for acoustically driven MZI, where a single SAW simultaneously modulates the refractive index of both MZI arms with opposite phases, as illustrated in Fig. 1(a). The opposite phase modulation of amplitude $\phi_{\max }$ per arm is achieved by simply spacing the WGs by an odd multiple of the half acoustic wavelength $\lambda_{\text {SAW }}$.

In this letter, we demonstrate an alternative concept for WG acousto-optical elements based on the modulation of several interferometer arms by a single SAW beam. These acousto-optic multiple interference devices (AOMIDs) provide integrated optics building blocks for functionalities such as switching, harmonic generation, and pulse shaping. ${ }^{7}$ The feasibility of the AOMID concept is established by the realization of a WG on/off switch for arbitrary time periods.

The AOMID concept is an extension of the acoustically driven MZIs of Fig. 1(a) for a number $N_{P} \geqslant 2$ of WG arms connected in parallel. ${ }^{8}$ The configurations for $N_{P}=2$ (corresponding to the simple MZI), $N_{P}=4$, and $N_{P}=8$ are illustrated in Figs. 1(a)-1(c). In each case, the single mode WGs (numbered by the index $p=1, \ldots, N_{P}$ ) are assumed to be identical and much narrower than $\lambda_{\mathrm{SAW}}$. The WGs are aligned perpendicularly to the SAW propagation direction and displaced laterally so as to experience SAW phases differing by a multiple of $\varphi_{\mathrm{SAW}}=2 \pi / N_{P}$. The amplitude $\phi_{\max }$ of the light phase shift upon propagation through the WGs is related to the WG length $\ell$ and to the refractive index modu-

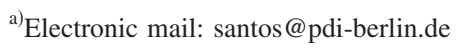

lation $\delta n$ induced by the SAW strain by $\phi_{\max }=2 \pi \delta n \ell / \lambda_{L}$, where $\lambda_{L}$ is the light wavelength. The use of symmetric Y-splitters in Fig. 1 ensures unity transmission in the absence of a SAW. Under the previous assumptions, the transmission $T_{P}$ of an AOMID with $N_{P}$ arms becomes:

$$
T_{P}^{\left(N_{P}\right)}[t]=\left|\frac{1}{N_{P}} \sum_{p=0}^{N_{P}-1} e^{i\left[\phi_{\max } \sin \left(2 \pi f_{\mathrm{SAW}}{ }^{t+p \varphi_{\mathrm{SAW}}}\right)\right]}\right|^{2},
$$

where $f_{\text {SAW }}$ is the SAW frequency. Figure 1(d) compares the time dependence of the transmission for AOMIDs with different number of arms. By an appropriate choice of $\phi_{\max }$, the transmission becomes strongly suppressed when the SAW is turned on, thus illustrating the operation as a switch for arbitrary on/off times.

The transmission suppression can be intuitively understood by taking into account that for symmetric devices (as the ones in Fig. 1) (i) any variation in the refractive index leads to a reduced transmission and that (ii) the transmission is invariant under shifts of the SAW phase by multiples of

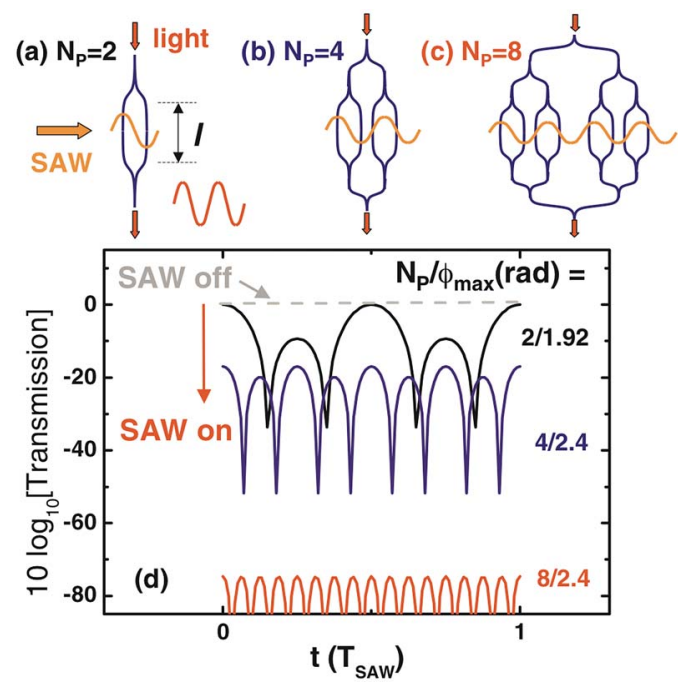

FIG. 1. (Color online) Acousto-optical multiple interference devices (AOMIDs) with (a) $N_{P}=2$, (b) $N_{P}=4$, and (c) $N_{P}=8$ arms. (d) Transmission for the given phase modulation $\phi_{\max }$ for AOMID with $N_{P}=2\left(\phi_{\max }=1.92 \mathrm{rad}\right)$, $N_{P}=4\left(\phi_{\max }=2.4 \mathrm{rad}\right)$, and $N_{P}=8\left(\phi_{\max }=2.4 \mathrm{rad}\right)$. 


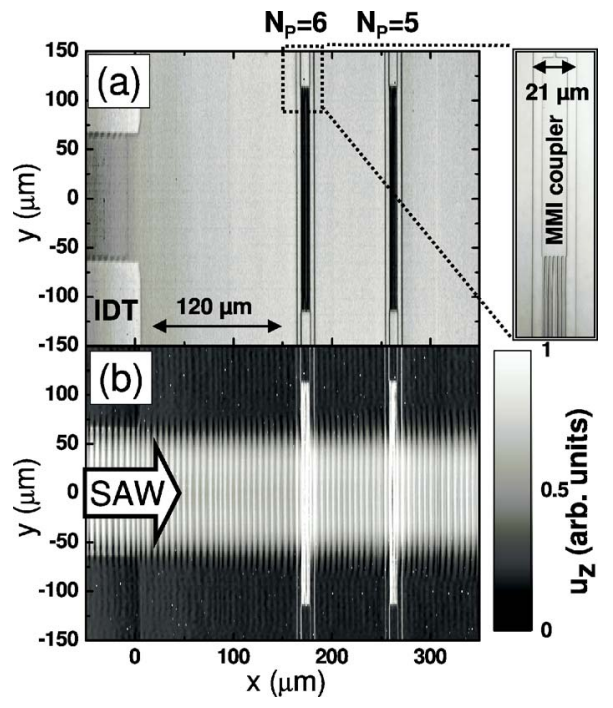

FIG. 2. (Color online) (a) Optical and (b) interferometry micrographs of the vertical component $u_{z}$ of the acoustic field of structures containing five- and sixfold AOMIDs. The inset shows the MMI coupler for the sixfold AOMID.

$2 \pi / N_{P}$ [cf. Fig. 1(a) -1 (c)]. As a result, when the refractive index of the arms is modulated by a SAW with frequency $f_{\mathrm{SAW}}$, the transmission will reduce $2 N_{P}$ times within a SAW cycle and only contains harmonics of the frequency $N_{P} f_{\text {SAW }}$. In fact, it can be shown that in the limit $N_{P} \rightarrow \infty$ the transmission becomes proportional to $J_{0}^{2}\left(\phi_{\max }\right){ }^{7}$ where $J_{0}$ is the zero-order Bessel function, which vanishes for $\phi_{\max }=2.405 \mathrm{rad}$.

In order to demonstrate the previous concepts, we have fabricated AOMIDs with $N_{P}=2-6$ on an (Al, Ga)As WG sample grown on GaAs (100) by molecular beam epitaxy. The sample consists of a 300-nm-thick GaAs film forming the core of a surface WG deposited on a 1500-nm-thick $\mathrm{Al}_{0.2} \mathrm{Ga}_{0.8} \mathrm{As}$ cladding layer. AOMIDs with $N_{P}=2$ and 4 were designed using symmetric Y-splitters to couple the input and output WGs to the 700-nm-wide WG arms. The devices with $N_{P}=3,5$, and 6 employed multimode interference (MMI) couplers. ${ }^{9}$ In this case, the width of the individual WGs was adjusted to ensure the same overall light phase shift through each WG arm. The AOMIDs were fabricated in two steps using contact optical lithography. First, the 150-nm-deep grooves delimiting the ridge WGs were produced by plasma etching. Subsequently, interdigital transducers (IDTs) for SAW generation were fabricated using a lift-off metalization process to structure a 60 -nm-thick metal layer. The splitfinger IDTs have an aperture of $120 \mu \mathrm{m}$ and were designed for $\lambda_{\mathrm{SAW}}=5.6 \mu \mathrm{m}$ (corresponding to $f_{\mathrm{SAW}} \approx 520 \mathrm{MHz}$ ). The radio-frequency (rf) power $P_{\mathrm{rf}}=V_{\mathrm{rf}}^{2} / R_{50}\left(R_{50}=50 \Omega\right)$ applied to the IDTs will be specified in terms of the nominal rms voltage $V_{\mathrm{rf}}$. The fraction $r_{p}=0.14 \pm 0.03$ of $P_{\mathrm{rf}}$ converted to the acoustic mode was determined from rf-reflection measurements on the IDTs. Figure 2(a) shows a micrograph of the sample region with AOMIDs with $N_{P}=5$ and $N_{P}=6$, together with details of the MMI for the $N_{P}=6$ structure (inset). Note that both AOMIDs are driven by the same SAW beam.

An important consideration for AOMIDs regards the distribution of the acoustic fields across the WG structure, which was measured using a microscopic scanning Michelson interferometer. The two-dimensional plot of Fig. 2(b) displays the amplitude of the surface displacement $u_{z}$ reDownloaded 06 Nov 2007 to 147.156.161.82. Redistribution subject

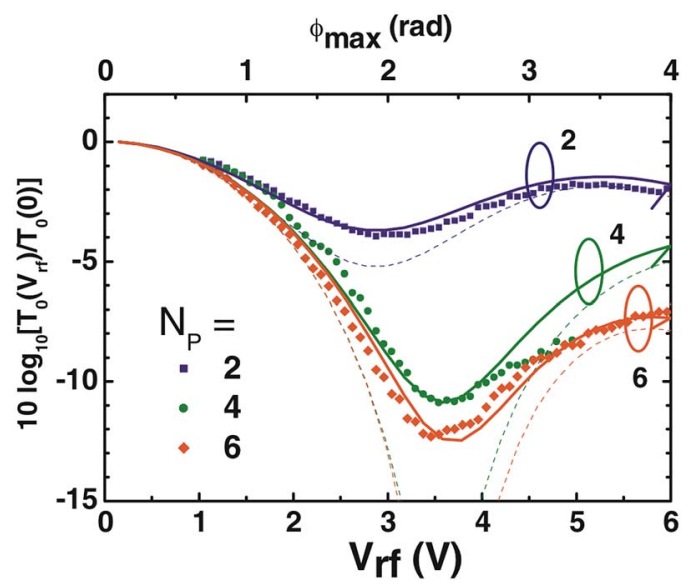

FIG. 3. (Color online) Symbols: Average (dc) transmission $T_{0}$ as a function of the rms voltage $\left(V_{\mathrm{rf}}\right)$ applied to the IDT for AOMIDs with $N_{P}=2$ (measured at $\left.\lambda_{L}=910 \mathrm{~nm}\right), N_{P}=4\left(\lambda_{L}=890 \mathrm{~nm}\right)$, and $N_{P}=6\left(\lambda_{L}=900 \mathrm{~nm}\right)$. The dashed and solid lines display the calculated transmission for devices with symmetric and slightly asymmetric arms, respectively.

corded in the same area as in Fig. 2(a) for a particular SAW phase. The SAW wave fronts appear as finely spaced vertical lines separated by $\lambda_{\mathrm{SAW}}=5.6 \mu \mathrm{m}$. The SAW beam width corresponds closely to the aperture of the IDT of $120 \mu \mathrm{m}$ and shows negligible attenuation (less than 5\%) after traversing the five- and six-fold AOMIDs. The low attenuation is associated with the small ratio between the depth of the WG grooves and $\lambda_{\mathrm{SAW}}$ and clearly demonstrates that a single SAW beam can efficiently drive AOMIDs with a large number of arms.

The devices were optically characterized by coupling light into the input WG using a tapered fiber with a cylindrical lens on its tip. As light source, we used a superluminescent diode with a 50-nm-wide emission peak centered at the wavelength $\lambda=920 \mathrm{~nm}$. The fiber was mounted on a piezoelectrically controlled stage in order to allow for a fine positioning relative to the WG edge. The transmitted light was collected using a microscope objective $(50 \times)$ focused on the cleaved edge of the output WG and detected using an optical spectrometer.

The operation of AOMIDs as on/off switches is demonstrated in Fig. 3, which displays the time-averaged transmission $T_{0}\left(V_{\mathrm{rf}}\right)$ (normalized to the corresponding value in the absence of acoustic excitation) for devices with different numbers of arms. The light wavelength was slightly changed for the different devices in order to enhance the on/off contrast ratio by partially compensating for asymmetries in the WGs (see below). For $N_{P}=2$ (corresponding to a simple MZI), $T_{0}$ reaches a minimum of $-6 \mathrm{~dB}$ for $V_{\mathrm{rf}}=2.8 \mathrm{~V}$. The minimum transmission shifts to higher $V_{\mathrm{rf}}$ and reduces with $N_{P}$ down to $-12 \pm 1 \mathrm{~dB}$ for the sixfold device. The dashed lines show the dependence of $T_{0}$ on $\phi_{\max }$ (upper scale) calculated for symmetric devices using a SAW-induced refractive index $\delta n\left(V_{\mathrm{rf}}\right)=r_{n} V_{\mathrm{rf}} \sqrt{r_{p} / 2 R_{50} \ell}$. Here, the factor 2 in the denominator accounts for the bidirectionality of the IDT, i.e., the fact that the IDT emits both along the positive and negative $x$ directions. The acousto-optical coupling factor $r_{n}$ was obtained from the photoelastic coefficients of GaAs (Ref. 10) and from the the depth dependence of the SAW strain fields. ${ }^{11}$ Further details about the calculations will be given in Ref. 7. In order to fit the position of the minima, we use photoelastic coefficients $15 \%$ higher than those reported for to AIP license or copyright, see http://apl.aip.org/apl/copyright.jsp 


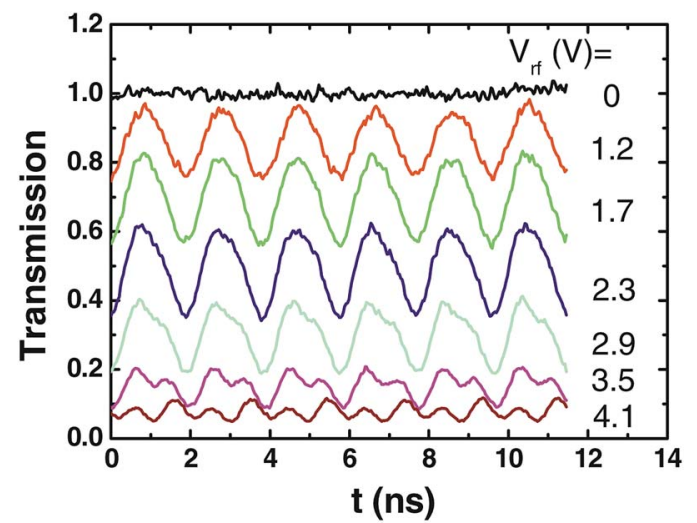

FIG. 4. (Color online) Time-resolved traces of the periodic transmission of a sixfold AOMID measured at $\lambda_{L}=910 \mathrm{~nm}$ for different rf-voltages $\left(V_{\mathrm{rf}}\right)$ applied to the IDT.

$\lambda=1 \mu \mathrm{m} .{ }^{10}$ The latter is attributed to the enhanced acoustooptical coupling close to the GaAs gap.

The calculations reproduce well the voltage dependence of the transmission for small phase amplitudes $\phi_{\max }$. For phases near $2.4 \mathrm{rad}$, however, they predict a much stronger suppression of the transmission than the ones measured for devices with $N_{P} \geqslant 4$. In order to address this discrepancy, we have carried out time-resolved (time resolution of $600 \mathrm{ps}$ ) transmission measurements by selecting a narrow $(10 \mathrm{~nm})$ spectral band and detecting the light using an avalanche photodiode. Figure 4 shows time-resolved transmission traces recorded on an AOMID with six arms for different $V_{\text {rf }}$. In agreement with Fig. 3, the average transmission reduces with $V_{\mathrm{rf}}$ and reaches a minimum of approximately $5 \%(=13 \mathrm{~dB})$ for $V_{\mathrm{rf}}=4 \mathrm{~V} .{ }^{12}$ The transmission reduction is accompanied by pronounced oscillations at the first (for $1.2<V_{\mathrm{rf}}<2.9 \mathrm{~V}$ ) and second harmonics (for $2.9 \mathrm{~V}<V_{\text {rff }}$ ) of the SAW frequency. These harmonics, which are not expected for symmetric AOMIDs, are attributed to asymmetries of the WG arms arising from differences in the optical length $(\delta \ell)$ or transmission of the individual WGs. In order to illustrate this behavior, the solid lines in Fig. 3 were calculated assuming a maximum path difference $\delta \ell_{\max }^{(\mathrm{opt})}=140 \mathrm{~nm}$ between any of the arms. For devices with $N_{P}>2$, there are different ways of distributing the path differences between the arms. In the calculations, we simply assumed that it increases linearly from 0 to $\delta \ell_{\max }^{\text {(opt) }}$ with the distance from the transducer. This simple model reproduces reasonably well the transmission over a wide range of phases. Since $\delta \ell_{\max }^{(\mathrm{opt})} / n \sim 40 \mathrm{~nm}$ is comparable to the tolerances of the employed optical lithography, much higher on/off contrast ratios are expected for an improved fabrication process.

In conclusion, we have demonstrated the feasibility of the AOMID concept in realizing an on/off switch with a high contrast ratio.

The authors thank K.-F. Friedland for comments and for critical reading of the letter as well as M. Höricke, H. Kostial, and A. Scheu for the preparation of the devices. Support from the EU Network of Excellence ePIXnet is gratefully acknowledged.

${ }^{1}$ A. Korpel, Acousto-Optics (Dekker, New York, 1997).

${ }^{2}$ W. L. Liu, P. S. J. Russell, and L. Dong, Opt. Lett. 22, 1515 (1997).

${ }^{3}$ P. V. Santos, J. Appl. Phys. 89, 5060 (2001).

${ }^{4}$ C. Gorecki, E. Bonnotte, H. Toshioshi, F. Benoit, H. Kawakatsu, and H. Fujita, Proc. SPIE 3098, 392 (1997).

${ }^{5}$ C. Gorecki, F. Chollet, E. Bonnotte, and H. Kawakatsu, Opt. Lett. 22, 1784 (1997).

${ }^{6}$ M. M. de Lima, Jr., M. Beck, R. Hey, and P. V. Santos, Appl. Phys. Lett. 89, 121104 (2006).

${ }^{7}$ M. Beck, M. M. de Lima, Jr., and P. V. Santos, J. Appl. Phys. (to be published).

${ }^{8}$ Similar functionalities can be achieved by connecting several interferometer arms in series, see Ref. 7.

${ }^{9}$ L. B. Soldano, F. B. Veerman, M. K. Smit, B. H. Verbeek, A. H. Dubost, and E. C. M. Pennings, J. Lightwave Technol. 10, 1843 (1992).

${ }^{10}$ Elastic, Piezoelectric, Pyroelectric, Piezooptic, Electrooptic Constants and Nonlinear Dielectric Susceptibilities of Crystals, Landolt-Börnstein Vol. 11, edited by K. H. Hellwege and A. M. Hellwege (Springer, Heidelberg, 1979).

${ }^{11}$ M. M. de Lima, Jr. and P. V. Santos, Rep. Prog. Phys. 68, 1639 (2005).

${ }^{12} V_{\text {rf }}$ for minimum transmission is higher than in Fig. 3 due to the lower efficiency of the IDT. 